OPEN

SUBJECT AREAS:

CHARACTERIZATION AND ANALYTICAL

TECHNIQUES

WETTING

Received

25 June 2014

Accepted

5 August 2014

Published

30 September 2014

Correspondence and requests for materials should be addressed to W.J. (whihe@snu.ac.

$\mathrm{kr})$

* Current address: National Institute of Standards and Technology,

Gaithersburg, MD 20899, USA.

\section{Energy dissipation of nanoconfined hydration layer: Long-range hydration on the hydrophilic solid surface}

\author{
Bongsu Kim, Soyoung Kwon, Hyosik Mun, Sangmin An* \& Wonho Jhe
}

Department of Physics and Astronomy, Institute of Applied Physics, Seoul National University, Seoul 151-747, Korea.

The hydration water layer (HWL), a ubiquitous form of water on the hydrophilic surfaces, exhibits anomalous characteristics different from bulk water and plays an important role in interfacial interactions. Despite extensive studies on the mechanical properties of HWL, one still lacks holistic understanding of its energy dissipation, which is critical to characterization of viscoelastic materials as well as identification of nanoscale dissipation processes. Here we address energy dissipation of nanoconfined HWL between two atomically flat hydrophilic solid surfaces (area of $\sim 120 \mathrm{~nm}^{2}$ ) by small amplitude-modulation, noncontact atomic force microscopy. Based on the viscoelastic hydration-force model, the average dissipation energy is $\sim 1 \mathrm{eV}$ at the tapping amplitude $(\sim 0.1 \mathrm{~nm})$ of the tip. In particular, we determine the accurate $\mathrm{HWL}$ thickness of $\sim 6$ layers of water molecules, as similarly observed on biological surfaces. Such a long-range interaction of HWL should be considered in the nanoscale phenomena such as friction, collision and self-assembly.

T he hydration water layer (HWL) is a universal thin film of water formed on the hydrophilic surfaces in ambient conditions or in aqueous solutions ${ }^{1}$, whose characteristics is critical to better understanding of numerous HWL-related phenomena such as interfacial adhesion ${ }^{2}$, friction between surfaces ${ }^{3,4}$, filtration ${ }^{5}$, molecular transport in nanostructures ${ }^{6,7}$, molecular assembly of particles in liquid water ${ }^{8,9}$, and even biological inter-cellular processes ${ }^{1}$. The common responsible mechanism may be associated with the unique nanoscale characteristics of HWL in contrast to bulk water, which have been measured by various methods. For example, the ordered water structure at the surface-liquid interface was observed by infrared spectroscopy ${ }^{10}$ or X-ray crystallography ${ }^{11}$. The inter- and intra-molecular dynamic motion have been analysed by terahertz spectroscopy and molecular dynamic simulation ${ }^{12-14}$. In particular, the mechanical anomalies of HWL confined in the nanometric gap and the relevant interfacial forces have been studied by surface force apparatus (SFA) ${ }^{1,4,15}$ and scanning force microscopy (SFM) ${ }^{2,3,16}$, including the largely enhanced viscosity ${ }^{3}$, nonlinear viscoelasticity ${ }^{17}$ and nonsqueeze-out fluidity ${ }^{4}$ of HWL.

Dissipation of energy is an important and critical physical process required for full characterization of nanoscale mechanics and dynamics of HWL. In general, the properties of energy dissipation are extensively analysed by the relevant force hysteresis ${ }^{2,18-24}$. However, it has been challenging to construct either the exact hydration-force hysteresis model due to the intricate hydration structure consisting of time-varying hydrogen-bond networks ${ }^{25,26}$, or the approximate force-hysteresis model due to the lack of the viscoelastic hydration-force formula. Without the explicit form of the hydration force, the elasticity and viscosity of HWL usually measured by atomic force microscopy (AFM) provide rather limited information for the energy dissipation of $\mathrm{HWL}^{3,16,17}$. Nonetheless, since the 'average' form of the viscoelastic hydration force is available ${ }^{16}$, one can address the overall response of the hydration structure by its first-order description of the energy dissipation of HWL, which can be done by performing measurements over the wide surface area of the AFM tip during detection time longer than relaxation time.

In this article, we first derive the explicit expression of the dissipated energy for the nanoconfined HWL based on the qualitative form of the viscoelastic hydration force ${ }^{16}$. We then demonstrate the validity of the calculated energy-dissipation formulas by comparing to the corresponding AFM experiments. In particular, it is remarkable that the quantitative energy dissipation analysis allows one to determine the exact thickness of HWL, beyond the well established information on the elastic and viscous properties of HWL. Experiments under various relative humidity $(\mathrm{RH})$ show that HWL consists of about 6 layers of water molecules on the hydrophilic solid surface. This 
is thicker than the results of both computer simulation ${ }^{27,28}$ and experiments performed by the AFM-based sharp tip ${ }^{25,26,29}$, where the results indicate the hydration layer effect on the solid surface disappears at about 3 layers.

\section{Results}

By using the quartz tuning-fork (QTF) based, small amplitudemodulation (AM) AFM in the tapping-mode operation (Figs. 1a, $1 \mathrm{~b}$ ), we have measured the mechanical properties of HWL confined between the flattened fused quartz tip (top right in Fig. 1c) and the mica substrate. The root mean squared (RMS) surface roughness of mica (Fig. 1d) and fused quartz rod (Fig. 1e) has been measured as $0.39 \AA$ and $0.14 \AA$, respectively, by a commercial AFM, which indicates that both surfaces are atomically flat (detailed information in Methods). Figure 2 presents the experimental effective elasticity $\left(k_{\text {int }}\right)$ and damping coefficient $\left(b_{\text {int }}\right)$ versus the distance $z_{0}$ between the tip and substrate. The contact point $\left(z_{\mathrm{c}}\right)$ is determined as the position where the standard deviation of the damping coefficient over repeated measurements abruptly increases by more than 5 times compared to that of the noncontact state as the tip approaches the substrate; $z_{\mathrm{c}}=0.3 \pm 0.3 \mathrm{~nm}$ which implies the fact that the monolayer of water molecules is not easily squeeze out in between the two flat hydrophilic surfaces.

Figures $3 \mathrm{a}, \mathrm{b}$ plot the effective elasticity $k_{\text {int }}$ of Fig. $2 \mathrm{a}$ in detail (Fig. 3a) and in semi-logarithmic scale (Fig. 3b) versus several values of RH. Notice that the measured $k_{\text {int }}$ combines the contributions of the nanoscale HWL as well as the capillary water-meniscus formed between the flattened fused quartz tip and the mica substrate. Figures $3 \mathrm{c}$, $3 \mathrm{~d}$ show the effective damping coefficient $b_{\text {int }}$ of Fig. $2 \mathrm{~b}$ in the semi-logarithmic scale. Interestingly, the humidity dependence of $k_{\text {int }}$ disappears when $z_{0}$ is less than $\sim 1.2 \pm 0.1 \mathrm{~nm}$ as shown in Fig. $3 \mathrm{~b}$, whereas the humidity dependence of $b_{\text {int }}$ appears above $\sim 3.3 \pm 0.5 \mathrm{~nm}$ in Fig. $3 \mathrm{c}$. The error values here are obtained by the standard deviation of the data obtained by repeated measurements.
The black lines in Fig. 3 represent the theoretical values of $k_{\mathrm{h}}$ and $b_{\mathrm{h}}$ that include only the contributions of HWL while excluding the effects of the capillary meniscus, given by ${ }^{16}$,

$$
k_{\mathrm{h}}=\frac{\Omega P_{0}}{\lambda_{0}} e^{-z_{0} / \lambda_{0}}, \quad b_{\mathrm{h}}=\frac{\Omega P_{0}}{v_{0}} e^{-z_{0} / \lambda_{0}},
$$

where $\Omega$ is the interaction area $\left(\pi r_{\mathrm{t}}^{2}\right)$ that is estimated as $120 \mathrm{~nm}^{2}$ from Fig. $1 \mathrm{~b}\left(r_{\mathrm{t}}\right.$ is the radius of the interaction area of the flattened tip). $v_{0}, P_{0}$ and $\lambda_{0}$ are the constant parameters associated with the velocity, pressure and characteristic length, obtained by curve fitting as $0.11 \pm 0.02 \mathrm{~mm} / \mathrm{s}, 1.78 \pm 0.08 \mathrm{MPa}$ and $1.01 \pm 0.06 \mathrm{~nm}$, respectively. Notice that $k_{\mathrm{h}}$ and $b_{\mathrm{h}}$ that result from HWL exhibit the decrease behaviour with the same characteristic length of the exponential decay as shown in Figs. 3b, 3d, which agree well to equation $(1)^{16}$.

Figures $4 \mathrm{a}, 4 \mathrm{~b}$ present the dissipated energy per tapping cycle $\left(E_{\mathrm{dis}}\right)$, versus the distance $z_{0}$ (Fig. $4 \mathrm{a}$ ) as well as versus the oscillation amplitude $A$ of the tip (Fig. 4b). The black curves in Figs. 4a, 4b represent $E_{\text {dis }}$ associated with $\mathrm{HWL}$, which is derived as follows,

$$
E_{\mathrm{h}}=2 A \lambda_{0} \pi \omega I_{1}\left(A / \lambda_{0}\right) \frac{\Omega P_{0}}{v_{0}} e^{-z_{0} / \lambda_{0}}
$$

where $I_{1}(x)$ is the modified Bessel function of the first kind. Notice that in Fig. $4 \mathrm{a}$, the experimental values of $E_{\text {dis }}$ deviate from equation (2) above $z_{0}$ in between $2.5 \mathrm{~nm}$ and $3.5 \mathrm{~nm}$, depending on $\mathrm{RH}$, which evidently indicates that the 'repulsive' effects of HWL disappear at the RH-dependent distance where the energy dissipation by the 'attractive' capillary force becomes dominant.

\section{Discussion}

When the two HWLs, one on the flattened tip and the other on the mica substrate, become close and contact each other over the wide surface area, they exert the 'average' repulsive force, that is, the positive pressure on both surfaces as shown by the positive values of $k_{\text {int }}$ a

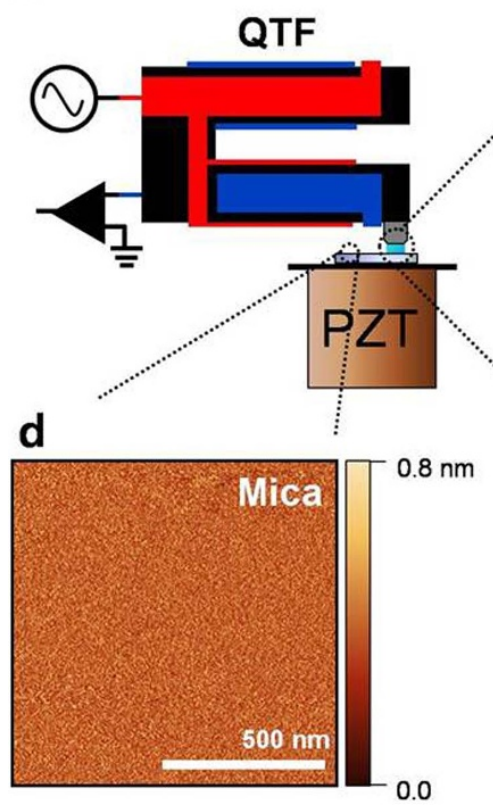

b

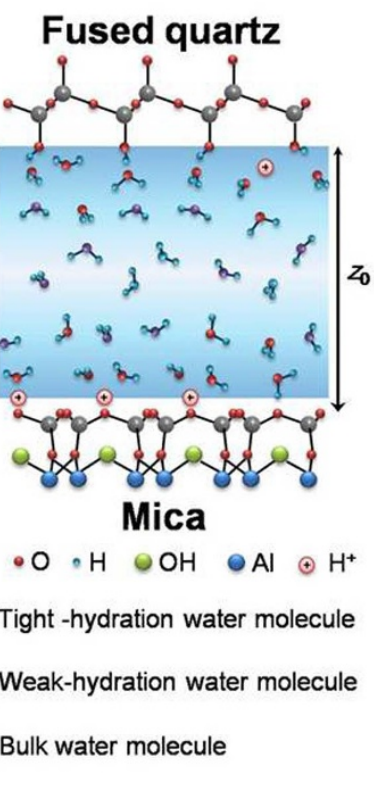

C
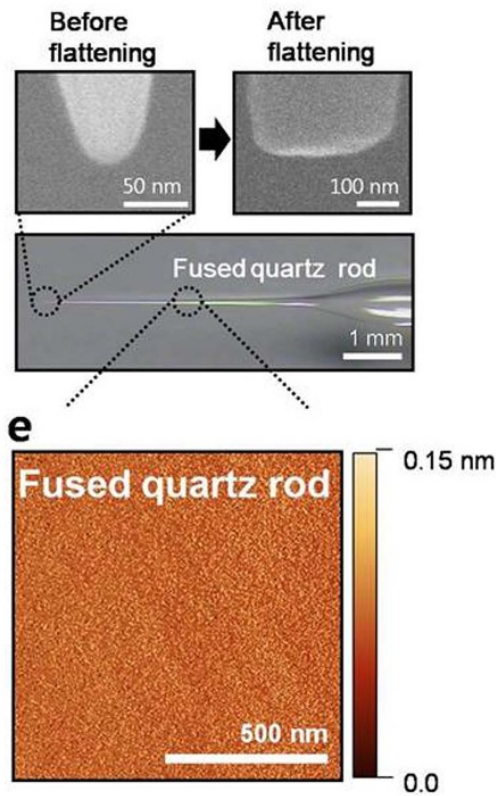

Figure 1 Experimental schematic and confined hydration water layer. (a) Experiments are performed using quartz tuning-fork (QTF) based AM-AFM. Mechanical properties of the confined hydration water layer (HWL) formed between the flattened fused quartz tip and mica surface are measured. (b) The water molecules are strongly hydrated close to the hydrophilic solid surfaces such as mica and quartz. Away from the surface, the molecules become increasingly weakly bound to the surface. (c) The fused quartz rod is fabricated by a mechanical laser puller (bottom) and the resulting sharp and round fused quartz tip is obtained (SEM image, top and left). The flattened tip used for experiments is obtained by repetitive gentle contacts to the mica surface (SEM image, top and right). (d) The root mean squared (RMS) surface roughness of mica is $0.39 \AA$ measured by a commercial AFM. (e) The RMS surface roughness of the fused quartz rod is $0.14 \AA$. Both the flattened tip and mica are shown to be atomically flat. 
a

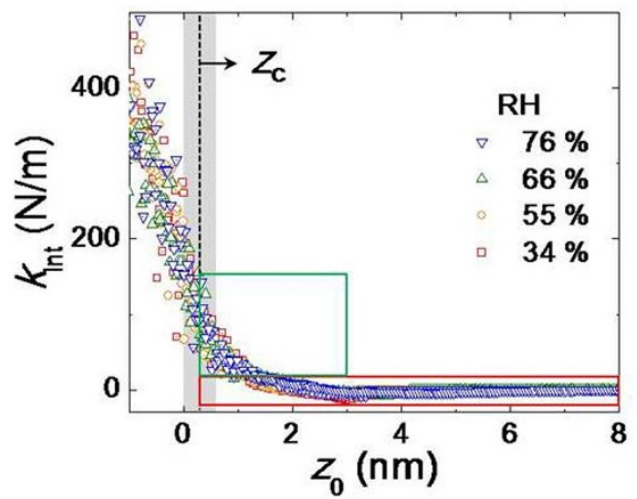

b

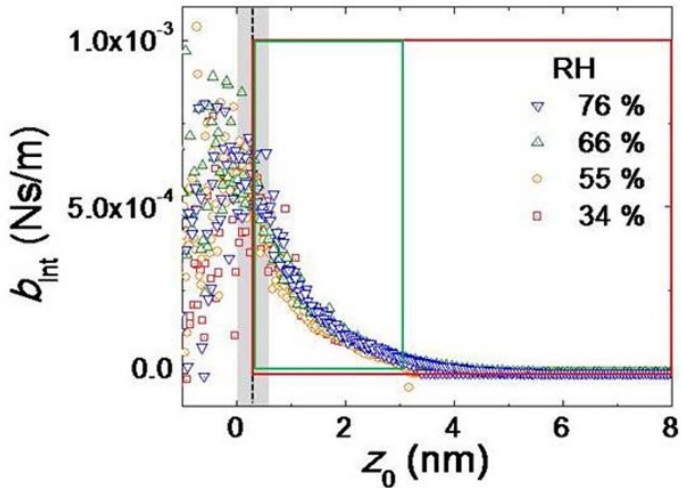

Figure 2 Experimental results and determination of contact point. (a) The measured elasticity and (b) damping coefficient resulting from the nanoconfined water (including the hydration water layer) between two atomically flat solid surfaces at various relative humidity (RH). The contact point $\left(z_{\mathrm{c}}\right)$ is determined as the position where the damping coefficient shows abrupt changes as the tip approaches the substrate: $z_{\mathrm{c}}=0.3 \pm 0.3 \mathrm{~nm}$ that corresponds to the single monolayer thickness of the hydration layer. The data in the red and green box are presented in detail in Fig. 3.

a
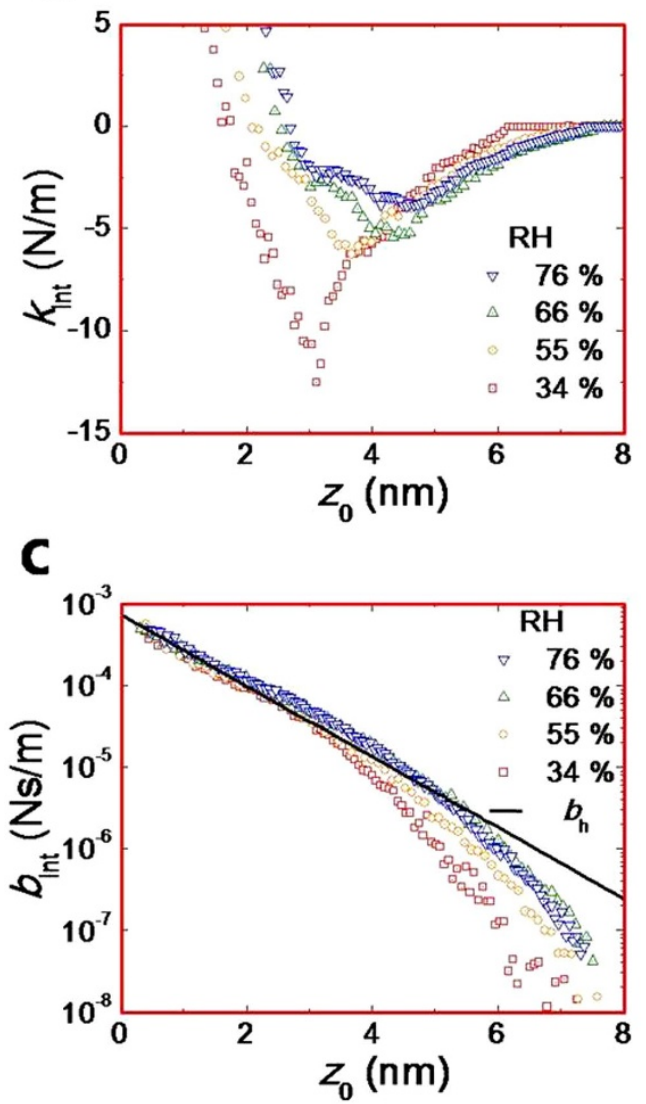

b

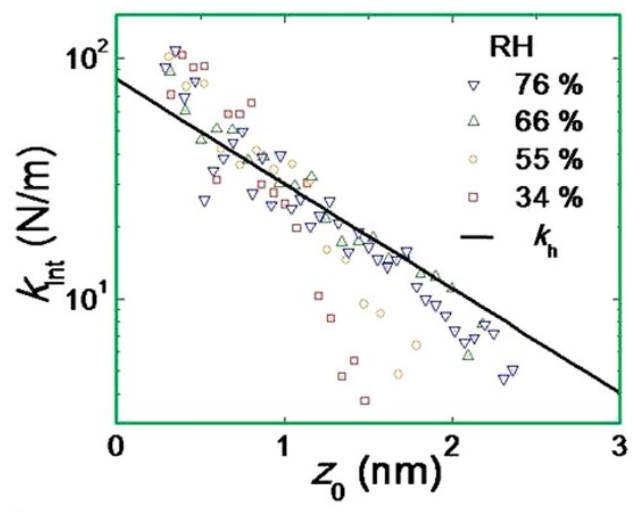

d

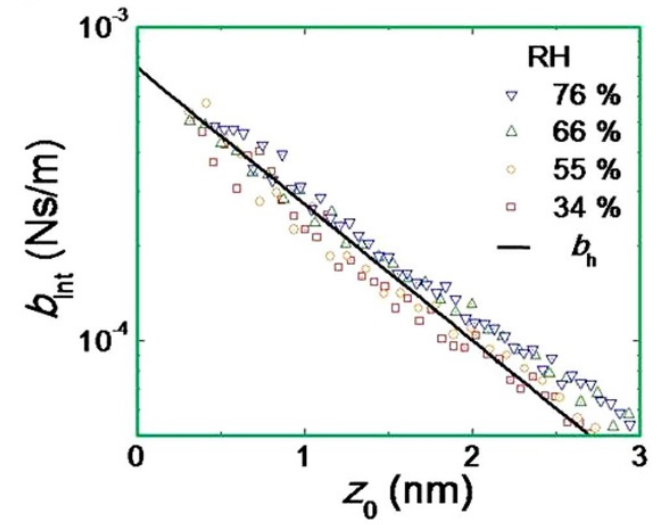

Figure 3 Variation of mechanical properties of the nanoconfined water. The data presented in the red (green) frame corresponds to the same data in the respective red (green) box in Fig. 2. (a), (b) Elasticity $\left(k_{\text {int }}\right)$ originates from the nanoconfined water that consists of both HWL and capillary nanomeniscus. (a) The humidity dependence appears where the distance $z_{0}$ is larger than $1.2 \mathrm{~nm}$, beyond which both the positive pressure due to the hydration layer and the negative Laplace pressure simultaneously contribute to $k_{\text {int }}$. $k_{\text {int }}$ becomes negative when the attractive Laplace pressure dominates at sufficiently large $z_{0}$. (d) Below $1.2 \mathrm{~nm}$ of $z_{0}, k_{\text {int }}$ is positive and is attributed to the strongly repulsive hydration force due to the tightly bound HWL, which is independent of RH. (c), (d) Damping coefficient $\left(b_{\text {int }}\right)$ associated with both HWL and capillary nanomeniscus. (c) The humidity dependence appears where $z_{0}$ is larger than $3.0 \mathrm{~nm}$. Unlike the case of elasticity, it is ambiguous to determine where the dissipative effect of the hydration layer dominates or where the capillary force-hysteresis effect contributes. (d) $b_{\text {int }}$ decreases with the same decay length as that of $k_{\text {int }}$, in good agreement to equation (1), indicating the effects of HWL. 

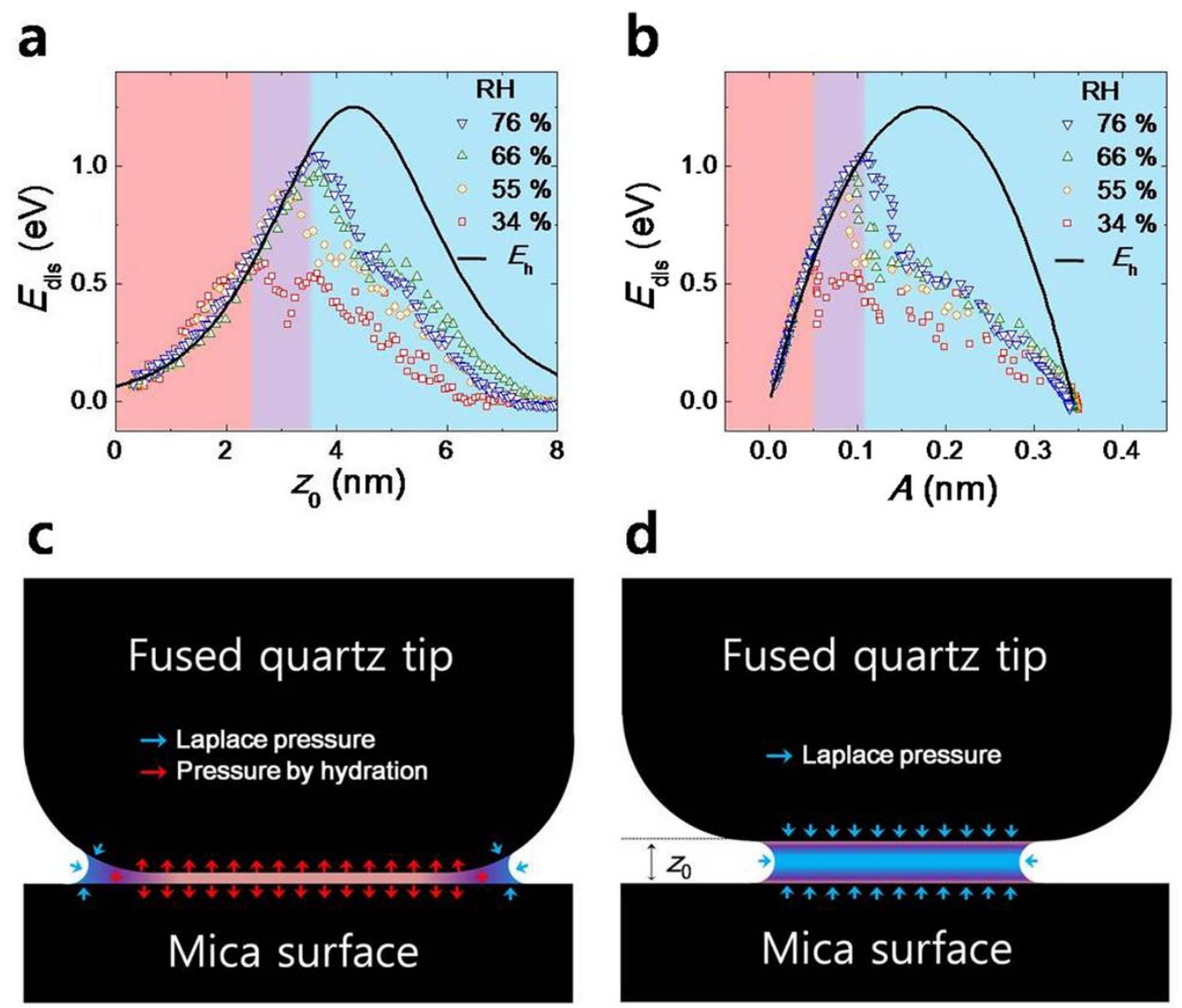

HWL dominant region Intermediate region

Capillarydominant region

Figure 4 Energy dissipation of HWL and capillary force. (a) Energy dissipation $\left(E_{\mathrm{dis}}\right)$ versus $z_{0}$. The black curve represents the theoretical values (equation (2)) obtained from the viscoelastic hydration-force model, which shows good agreement to the experimental results in the hydration region (including both the tightly and weakly bound HWL). The repulsive hydration layer is maintained up until about $3.5 \mathrm{~nm}$ of $z_{0}$ (that is, about 12 layer thickness of water molecules) at high RH. At low RH, the long-range hydration layer (or the weakly bound HWL) is collapsed by the attractive Laplace pressure effects, and thus only the tightly bound HWL remains. (b) $E_{\text {dis }}$ versus oscillation amplitude of tip. The black curve shows the theoretical results and is well fitted with the experimental results in the hydration region. Notice that the bell shape of $E_{\text {dis }}$ for the hydration layer exhibits the general behaviour similarly observed in other viscoelastic materials. (c) When the thickness of the nanoconfined water (including both HWL and capillary nanomeniscus) is sufficiently thin (under $\sim 2.5 \mathrm{~nm}$ of $z_{0}$ ), the tightly bound HWL formed on each surface exert positive (repulsive) pressure, as indicated by red arrows. Although the capillary meniscus may also exist at the rim of the tip, and provide negative (attractive) pressure (i.e., the Laplace pressure), its effect is dominated by the tightly bound HWL. (d) Above $3.5 \mathrm{~nm}$ of $z_{0}$, the Laplace pressure effect dominates with insignificant effects of HWL observed. The red, purple and blue colour in Fig. 4 represent the region of the tightly hydrated layer, weakly hydrated layer, and capillary nanomeniscus, respectively.

both in equation (1) and in Figs. $3 a, 3 b^{1,4,15,16,30}$. In particular, under $1.2 \mathrm{~nm}$ of $z_{0}$ (that is, within $\sim 4$ layers of water molecules), $k_{\text {int }}$ is positive (Fig. $3 \mathrm{~b}$ ) resulting from the repulsive pressure in good agreement to equation (1), and is independent of RH (see also Fig. 4c). When $z_{0}$ is larger than $\sim 1.2 \mathrm{~nm}$, on the other hand, $k_{\text {int }}$ shows the $\mathrm{RH}$-dependent deviation from equation (1) and becomes negative due to the attractive (capillary) force originating from the pressure difference, so called the Laplace pressure, between the interfacial liquid water and the surrounding vapour as shown in Fig. 3a (refer also to Fig. $4 \mathrm{~d})^{30-32}$. The Laplace pressure is the liquid-vapour surface tension divided by the radius of curvature of the water meniscus; $\Delta P$ $=\gamma / r_{\mathrm{k}}$, where $\gamma$ and $r_{\mathrm{k}}$ are the surface tension and radius of curvature, respectively ${ }^{30,32} \cdot r_{\mathrm{k}}$ corresponds to the thermodynamic Kelvin radius, which is closely connected to $\mathrm{RH}$ by the relation, $1 / r_{\mathrm{k}} \propto \ln (\mathrm{RH} /$ $100)^{30,32}$. Therefore, $\left|k_{\text {int }}\right|$ becomes larger (i.e., $\Delta P$ is larger) at the lower RH as shown in Fig. 3a (note that, however, $\left|k_{\text {int }}\right|$ increases with the increase of $\mathrm{RH}$ at $z_{0}$ beyond $4 \mathrm{~nm}$, which is attributed to the smaller size of the nanoconfined water bridge at the lower $\mathrm{RH}$ ). Although the Laplace pressure effects appear above $1.2 \mathrm{~nm}$ distance at the low RH, the small HWL effects still exist until being completely dominated by the capillary-force effects.

In Figs. 3c, 3d, $b_{\text {int }}$ also agrees with equation (1) at $z_{0}$ less than $3 \mathrm{~nm}$ (that is, within $\sim 10$ layers of water molecules), and the RH dependence appears above $3 \mathrm{~nm}$ distance. Notice that the capillary force associated with the Laplace pressure should also affect $b_{\text {int }}$ above $1.2 \mathrm{~nm}$, as is the case for $k_{\text {int }}$ (Figs. $\left.3 \mathrm{a}, 3 \mathrm{~b}\right)^{20}$. This additional effect on $b_{\text {int }}$ may be probable because the tip that undergoes small-amplitude oscillatory motion generally results in the appropriate force hysteresis in the dissipative system ${ }^{20}$. As shown in Fig. 3c, such an RH-dependent effect appears at $z_{0}$ above $3 \mathrm{~nm}$, where $b_{\text {int }}$ evidently deviates from equation (1). However, compared to the behaviours of $k_{\text {int }}$, we find the results of $b_{\text {int }}$ provide somewhat inconsistent information on the HWL characteristics as well as on the specific transition point from the HWL-dominating region to the capillary-dominating region. Therefore, this naturally leads one to investigate the energy dissipation process, in addition to $k_{\text {int }}$ and $b_{\text {int }}$, to have a full understanding of the dissipative property of HWL. 
The dissipation energy $\left(E_{\text {dis }}\right)$ presents how far the HWL effects reach above the surface, which cannot be clearly determined in Fig. 3, as already discussed. Figure 4 shows the plot of $E_{\text {dis }}$ versus $z_{0}$ and $A$ for several RH's. In Fig. $4 \mathrm{a}, E_{\text {dis }}$ is in excellent agreement to the theoretical values of equation (2) (black curve) under $\sim 2.5 \mathrm{~nm}$ of $z_{0}$ and is independent of RH, which corresponds to the HWL-dominating region that consists of the tightly bound HWL. However, beyond $\sim 3.5 \mathrm{~nm}$ distance, $E_{\text {dis }}$ deviates completely from the curve for all values of RH (almost saturating already at 76\%), which consists of the bulk water region where the capillary force effect dominates with no explicit effects of HWL (refer to previous discussions on $k_{\text {int }}$ and $\left.b_{\text {int }}\right)$. Notice that the black curve represents $E_{\mathrm{h}}$ or equation (2) that is derived from the viscoelastic hydration-force model where only the effects of HWL alone are taken into account. At the higher $\mathrm{RH}$, the distance where deviation occurs becomes farther, so that the 'effective' thickness of HWL is accordingly increased. However, it does not mean that all the water molecules in HWL are tightly bound at the high $\mathrm{RH}$ because HWL is formed only by the interaction between hydrophilic surface and water molecules, independently of the humidity. We attribute this intermediate region between 2.5 and $3.5 \mathrm{~nm}$ as resulting from 'collapse' of the effects of the weakly bound HWL due to the increasing Laplace pressure effect. In other words, in the intermediate region, some water molecules are maintained as weakly bound HWL while other water molecules behave similar to the bulk water. Thus, regardless of $\mathrm{RH}$, the measured $E_{\mathrm{dis}}$ is smaller than the theoretical value $E_{\mathrm{h}}$ that is obtained on the assumption that all the water molecules form the HWL. As the effect of the weakly bound HWL disappears, $E_{\text {dis }}$ deviates from equation (2) and thus becomes dependent on $\mathrm{RH}$ where $E_{\text {dis }}$ originates from the capillary force hysteresis as discussed in $b_{\text {int }}$.

The intriguing result is that the HWL exists thicker than usually expected. For example, at RH of $66 \%$ and $76 \%$, the HWL is maintained until $\sim 3.5 \mathrm{~nm}$ of $z_{0}$ that corresponds to about 12 layer thickness of water molecules. In other words, HWL is consisted of $\sim 6$ layer thickness $(\sim 1.75 \mathrm{~nm})$ of water molecules on each surface (that is, mica substrate and fused quartz tip), which includes the tightly as well as weakly bound HWL. The long range $(\sim 2.0 \mathrm{~nm})$ hydration effect that appears on the stiff and atomically flat solid surface has not been addressed to date, although similar effect has been recently measured on the biological surfaces by terahertz spectroscopy ${ }^{12-14}$. For example, computer simulations of HWL on the solid surface have suggested the water molecules above the third molecular layer already behave similarly to the bulk water ${ }^{27,28}$, and experiments that measured the structure of hydration layer on the mica surface using the AFM-based sharp tip have also indicated that the hydration effect disappears even under $1 \mathrm{~nm}^{25,26,29}$. On the other hand, the interfacial force experiments using SFA often measured that the force between two flat surfaces violates the DLVO (Derjaguin and Landau, Verwey and Overbeek) theory beyond $2 \mathrm{~nm}$ distance ${ }^{1,4,30}$. Here, our results unambiguously show that the long-range HWL is formed on the stiff and atomically flat solid surfaces like the biological samples. Figure $4 \mathrm{~b}$ presents $E_{\text {dis }}$ versus amplitude. Up until the collapse of the hydration effect, the theoretical model value (black curve) agrees well to the experimental data, and $E_{\text {dis }}$ versus amplitude shows the similar behaviours to other viscoelastic materials ${ }^{18,22}$. After the collapse, $E_{\text {dis }}$ results from the capillary force hysteresis ${ }^{20}$ and decreases along with the amplitude increase in contrast to the hydration effect.

In summary, we have derived the dissipation energy formulas associated with HWL from the simple viscoelastic hydration-force model, and provided its quantitative experimental study using the noncontact AM-AFM. The energy dissipation analysis helps determine the accurate range where HWL plays a dominant role, so that the thickness of HWL can be unambiguously measured. At high RH, the effect of HWL is maintained up to $3.5 \mathrm{~nm}$ thickness, which indicates the long-range hydration layer of about 6 layers of water molecules is formed on the solid surface. At low $\mathrm{RH}$, on the other hand, the Laplace pressure becomes strong and thus the long-range hydration layer collapses and only the tightly bound HWL remains below $\sim 2.5 \mathrm{~nm}$ distance, independently of $\mathrm{RH}$. Our results may provide new and significant insights for deeper understanding of the related phenomena, such as the nanoparticle self-assembly in ambient conditions. For example, the particle self-assembly process in air has been usually interpreted by the capillary interaction ${ }^{33-36}$ while the self-assembly of bio-molecule ${ }^{8}$ or nano-graphene ${ }^{9}$ in water has been understood dominantly by the HWL. However, when the particle size becomes nanometric scale, the interaction via the longrange HWL could be significant and thus should not be ignored. Moreover, it is also expected that the existence of the interplay between the long-range HWL and the Laplace pressure may contribute critically to collision as well as friction in the nanoscale systems such as the nanoelectromechanical system (NEMS) and colloidal nanoparticle system.

\section{Methods}

QTF-based AM-AFM. We measured the HWL properties between the fused quartz tip and mica using the quartz tuning-fork (QTF) based ${ }^{37,38}$ AM-AFM in the tappingmode operation ${ }^{39,40}$. The fused quartz tip is strongly epoxied to one prong of the QTF, which allows noncontact and sensitive force-gradient $(\sim 0.01 \mathrm{~N} / \mathrm{m}$; this is 10 times smaller than the measured smallest elasticity of nanoconfined water) measurement at variable height due to its high stiffness $(\sim 20,000 \mathrm{~N} / \mathrm{m})$ and quality factor $(\sim 10,000)$ at the resonant frequency $(\sim 32 \mathrm{kHz})$ in ambient conditions. Figure la is the schematic diagram of the QTF that has two separate terminals, where each terminal is connected to the electrodes on the QTF. The measured amplitude and phase signals of the tip are converted to the effective elasticity $\left(k_{\text {int }}\right)$, damping coefficient $\left(b_{\text {int }}\right)$ and energy dissipation per cycle $\left(E_{\text {dis }}\right)$ of the HWL between the two surfaces ${ }^{38,40}$. The equation of motion and the solution of the tip motion are given as follows,

$$
\begin{gathered}
m \ddot{z}+b \dot{z}+k z=F \cos \omega t-k_{\text {int }} z-b_{\text {int }} \dot{z}, \\
z=z_{0}+A(z) \sin [\omega t+\theta(z)], \quad v=A(z) \omega \cos [\omega t+\theta(z)],
\end{gathered}
$$

where $F$ is the amplitude of the driving force, $m, b, k$ and $\omega$ are the effective mass, damping coefficient, spring constant and driving angular frequency of QTF. And $z_{0}$, $A$ and $\theta$ are the mean separation between tip and substrate, oscillation amplitude and phase shift, respectively. Then $k_{\text {int }}, b_{\text {int }}$ and $E_{\text {dis }}$ are given by ${ }^{40}$,

$$
\begin{gathered}
k_{\text {int }}=\frac{F}{A(z)} \sin \theta(z)-k\left(1-\frac{\omega^{2}}{\omega_{0}^{2}}\right), \\
b_{\text {int }}=\frac{F}{A(z) \omega_{0}} \cos \theta(z)-b,
\end{gathered}
$$$$
E_{\mathrm{dis}}=\pi\left(F A \cos \theta(z)-b \omega A^{2}(z)\right),
$$

where $\omega_{0}$ is the resonance angular frequency of QTF. Notice that the trigonometric functions in equations (5), (6) and (7) should be adequately modified if different trigonometric functions are used in equations (3) and (4).

The flattened fused quartz tip that is attached to the QTF oscillates vertically at a given $z_{0}$ while $z_{0}$ itself is controlled by the piezoelectric transducer (PZT) on which the mica substrate is tightly fixed using an adhesive glue (Fig. 1a). Each data point shown in Figs. 2, 3, and 4 represent the time-average (for $300 \mathrm{~ms}$ ) values obtained using the lock-in amplifier. The prepared fused quartz tip and mica substrate are placed in an air-tight chamber which is filled with both dry and wet nitrogen gas. The relative humidity in the chamber is controlled by the mixture ratio between the two gases.

Fabrication of the flattened tip. The fabrication processes of the flattened fused quartz tip are as follows: (i) The sharp and round fused quartz tip (top and left in Fig. 1c) is fabricated by a laser-based mechanical puller (P-2000, Sutter Instruments Co.). We have measured the surface roughness of mica (Fig. 1d) and the fused quartz rod (Fig. 1e) by using contact-mode commercial AFM (Multimode SPM, Veeco Co.) where the diameter of the used cantilever tip is under $2 \mathrm{~nm}$ (SSS-NCLR-10,

Nanosensors Co.). The root mean squared (RMS) roughness of the mica and the fused quartz is $0.39 \AA$ and $0.14 \AA$, respectively. (ii) The round fused quartz tip is glued to one prong of the QTF using a strong epoxy (Araldite Rapid, Huntsman Advanced Materials Co.). (iii) The round fused quartz tip attached to the QTF is then forced to undergo repetitive, gentle contact against the atomically flat mica surface several hundred times. During this process, the round fused quartz tip becomes flattened. Then, without changing the alignment of the experimental setup, we perform experiments at several positions on the mica. The SEM image (top right of Fig. 1c) of the flattened tip was obtained after performing experiments. Notice that although the SEM image does not provide the nanometric details of the surface roughness of the flattened tip used in our experiments, it is still expected to exhibit the same 
subnanometric surface roughness, as indicated by the AFM image on the side surface of the fused quartz (Fig. 1e).

Theoretical model of energy dissipation. The energy dissipated by an oscillating system is defined by,

$$
E_{\mathrm{dis}}=\int_{T} v F_{\mathrm{nc}} d t
$$

where $F_{\mathrm{nc}}$ is the dissipative force, $v$ the velocity and $T$ one period of the oscillator motion. The non-conservative dissipation force of the viscoelastic hydration model is given by ${ }^{16}$

$$
F_{\mathrm{nc}}=\Omega P_{0} \frac{v}{v_{0}} e^{-z / \lambda_{0}},
$$

where $\Omega$ is the interaction area, $v_{0}, P_{0}$ and $\lambda_{0}$ are the constants associated with velocity, pressure and characteristic length, respectively. Inserting equation (9) into equation (8), using equation (4) as the position and velocity of the oscillator, and then integrating over an oscillation period, one can derive the energy dissipation per period as given in equation (2). The constants $\left(v_{0}, P_{0}\right.$ and $\left.\lambda_{0}\right)$ in equation (2) can be obtained by fitting the experimental $k_{\text {int }}$ and $b_{\text {int }}$ data to the effective elasticity $\left(k_{\mathrm{h}}\right)$ and damping coefficient $\left(b_{\mathrm{h}}\right)$ for the viscoelastic hydration force model ${ }^{16}$. Since $k_{\text {int }}$ and $b_{\text {int }}$ in equations (5) and (6) are matched to equation (1) by fitting, the amplitude $A$ can be obtained from equations (5) and (6) as,

$$
A=\frac{F}{\sqrt{\left(k_{\mathrm{h}}+\Delta k\right)^{2}+\left(b_{\mathrm{h}}+b\right)^{2} \omega^{2}}},
$$

where $\Delta k=k\left(1-\omega^{2} / \omega_{0}^{2}\right)$ is the detuning (In supplementary information, the theoretical values (equation (10)) are compared to the experimental data.) As a result, one can obtain analytically the dissipation energy of HWL using the viscoelastic hydration force model.

1. Israelachvili, J. \& Wennerström, H. Role of hydration and water structure in biological and colloidal interactions. Nature 379, 219-225 (1996).

2. Voitchovsky, K., Kuna, J., Contera, S., Tosatti, E. \& Stellacci, F. Direct mapping of the solid-liquid adhesion energy with subnanometre resolution. Nat. Nanotech. 5 , 401 (2010).

3. Ortiz-Young, D., Chiu, H., Kim, S., Voitchovsky, K. \& Riedo, E. The interplay between apparent viscosity and wettability in nanoconfined water. Nat. Commun. 4, 2482, doi: 10.1038/ncomms3482 (2013).

4. Raviv, U. \& Klein, J. Fluidity of bound hydration layers. Science 297, 1540 (2002).

5. Huang, H. et al. Ultrafast viscous water flow through nanostrand-channelled graphene oxide membranes. Nat. Commun. 4, 2979, doi: 10.1038/ncomms3979 (2013).

6. Chiavazzo, E., Fasano, M., Asinari, P. \& Decuzzi, P. Scaling behaviour for the water transport in nanoconfined geometries. Nat. Commun. 5, 3565, doi: 10.1038/ ncomms4565 (2014).

7. Duan, C. \& Majumdar, A. Anomalous ion transport in 2-nm hydrophilic nanochannels. Nat. Nanotech. 5, 848-852 (2010).

8. Ahmad, M., Gu, W., Geyer, T. \& Helms, V. Adhesive water networks facilitate binding of protein interfaces. Nat. Commun. 2, 261, doi: 10.1038/ncomms1258 (2011).

9. Lv, W. \& Wu, R. The interfacial-organized monolayer water film (MWF) induced "two-step" aggregation of nanographene: both in stacking and sliding assembly pathways. Nanoscale 5, 2765 (2013).

10. Cantrell, W. \& Ewing, G. Thin film water on muscovate mica. J. Phys. Chem. 105, 5434-5439 (2001).

11. Burling, F., Weis, W., Flaherty, K. \& Brünger, A. Direct Observation of Protein Solvation and Discrete Disorder with Experimental Crystallographic Phases. Science 271, 72-77 (1996).

12. Ebbinghaus, S. et al. An extended dynamical hydratoin shell around protein. Proc. Natl. Acad. Sci. 104, 20749-20752 (2007).

13. Hishida, M. \& Tanaka, K. Long-range hydration effect of lipid membrane studied by terahertz time-domain spectroscopy. Phys. Rev. Lett. 106, 158102 (2011).

14. Meister, K. et al. Long-range protein-water dynamics in hyperactive insect antifreeze proteins. Proc. Natl. Acad. Sci. 110, 1617-1622 (2013).

15. Zhu, Y. \& Granick, S. Viscosity of interfacial water. Phys. Rev. Lett. 87, 096104 (2001).

16. Kim, B. et al. Unified stress tensor of the hydration water layer. Phys. Rev. Lett. 111, $246102(2013)$

17. Li, T. \& Riedo, E. Nonlinear viscoelastic dynamics of nanoconfined wetting liquids. Phys. Rev. Letts. 100, 106102 (2008).

18. García, R. et al. Identification of nanoscale dissipation processes by dynamic atomic force microscopy. Phys. Rev. Lett. 97, 016103 (2006).

19. Martínez, N. \& García, R. Measuring phase shifts and energy dissipation with amplitude modulation atomic force microscopy. Nanotechnology 17, S167-S172 (2006).

20. Sahagún, E., García-Mochales, P., Sacha, G. \& Sáenz, J. Energy dissipation due to capillary interactions: hydrophobicity maps in force microscopy. Phys. Rev. Lett. 98, 176101 (2007).
21. Hofbauer, W. et al. Crystalline structure and squeeze-out dissipation of liquid solvation layers observed by small-amplitude dynamic AFM. Phys. Rev. B $\mathbf{8 0}$, 134104 (2009).

22. Gómez, C. \& García, R. Determination and simulation of nanoscale energy dissipation processes in amplitude modulation AFM. Ultramicroscopy 110, 626-633 (2010).

23. Santos, S. et al. Energy dissipation distributions and dissipative atomic processes in amplitude modulation atomic force microscopy. Nanotechnology 23, 125401 (2012).

24. Cartagena, A., Hernando-Pérez, M., Carrascosa, J., Pablo, P. \& Raman, A. Mapping in vitro local material properties of intact and disrupted virions at high resolution using multi-harmonic atomic force microscopy. Nanoscale $\mathbf{5}$, 4729-4736 (2013).

25. Fukuma, T., Ueda, Y., Yoshioka, S. \& Asakawa, H. Atomic-Scale Distribution of Water Molecules at the Mica-Water Interface Visualized by Three-Dimensional Scanning Force Microscopy. Phys. Rev. Lett. 104, 016101 (2010).

26. Herruzo, E., Asakawa, H., Fukuma, T. \& García, R. Three-dimensional quantitative force maps in liquid with 10 piconewton, angstrom and sub-minute resolutions. Nanoscale 5, 2678-2685 (2013).

27. Leng, Y. \& Cummings, P. Hydration structure of water confined between mica surfaces. J. Chem. Phys. 124, 074711 (2006).

28. Malani, A., Ayappa, K. \& Murad, S. Influence of hydrophilic surface specificity on the structural properties of confined water. j. Phys. Chem. B 113, 13825-13839 (2009).

29. Labuda, A., Kobayashi, K., Suzuki, K., Yamada, H. \& Grüter, P. Monotonic damping in nanoscopic hydration experiments. Phys. Rev. Lett. 110, 066102 (2013).

30. Israelachvili, J. Intermolecular and surface forces (Academic, San Diego, 2011).

31. Kwon, S., Stambaugh, C., Kim, B., An, S. \& Jhe, W. Dynamic and static measurement of interfacial capillary forces by a hybrid nanomechanical system. Nanoscale 6, 5474-5478 (2014)

32. Köber, M. et al. Nanogeometry Matters: Unexpected decrease of capillary adhesion forces with increasing relative humidity. Small 6, 2725-2730 (2010)

33. Yunker, P., Still, T., Lohr, M. \& Yodh, A. Suppression of the coffee-ring effect by shape-dependent capillary interactions. Nature 476, 308-311 (2011).

34. Gallego-Gómez, F., Morales-Flórez, V., Blanco, A., Rosa-Fox, N. \& López, C. Water- Dependent Micromechanical and Rheological Properties of Silica Colloidal Crystals Studied by Nanoindentation. Nano Lett. 12, 4920-4924 (2012).

35. Ershov, D. et al. Capillarity-induced ordering of spherical colloids on an interface with anisotropic curvature. Proc. Natl. Acad. Sci. 110, 9220-9224 (2013).

36. Colosqui, C. \& Morris, J. Hydrodynamically Driven Colloidal Assembly in Dip Coating. Phys. Rev. Lett. 110, 188302 (2013).

37. Giessibl, F. Advances in atomic force microscopy. Rev. Mod. Phys. 75, 949-983 (2003).

38. Lee, M., Jahng, J., Kim, K. \& Jhe, W. Quantitative atomic force measurement with a quartz tuning fork. Appl. Phys. Lett. 91, 023117 (2007).

39. García, R. \& Pérez, R. Dynamic atomic force microscopy methods. Surf. Sci. Rep. 47, 197-301 (2002)

40. Lee, M. \& Jhe, W. General theory of amplitude-modulation atomic force microscopy. Phys. Rev. Lett. 97, 036104 (2006).

\section{Acknowledgments}

This work was supported by the National Research Foundation of Korea (NRF) grant funded by the Korea government (MSIP) (No. 200983512).

\section{Author contributions}

B.K. and W.J. designed and directed the research. B.K., S.K. and W.J. wrote the manuscript. B.K. and S.K. prepared the samples and tips, and carried out the experiments. B.K. and W.J. developed the theoretical model of the energy dissipation process due to the hydration layer. H.M. measured the roughness of mica and fused quartz rod. S.A. helped experiment and provided invaluable discussions.

\section{Additional information}

Supplementary information accompanies this paper at http://www.nature.com/ scientificreports

Competing financial interests: The authors declare no competing financial interests.

How to cite this article: Kim, B., Kwon, S., Mun, H., An, S. \& Jhe, W. Energy dissipation of nanoconfined hydration layer: Long-range hydration on the hydrophilic solid surface. Sci. Rep. 4, 6499; DOI:10.1038/srep06499 (2014).

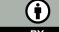

This work is licensed under a Creative Commons Attribution 4.0 International License. The images or other third party material in this article are included in the article's Creative Commons license, unless indicated otherwise in the credit line; if the material is not included under the Creative Commons license, users will need to obtain permission from the license holder in order to reproduce the material. To view a copy of this license, visit http://creativecommons.org/licenses/by/4.0/ 